\title{
Denosumab Reduces the Incidence of New Vertebral Fractures in Men With Prostate Cancer
}

\author{
David S. Silver
}

Published online: 19 February 2010

(C) The Author(s) 2010. This article is published with open access at Springerlink.com

\section{Trial}

Smith MR, Egerdie B, Hernandez Toriz N, et al.: Denosumab in men receiving androgen-deprivation therapy for prostate cancer. $N$ Engl J Med 2009, 361:745-765.

\section{Rating \\ -Of importance.}

\section{Introduction}

Prostate cancer is the most commonly diagnosed cancer among men worldwide. Androgen deprivation therapy has been a well established treatment for prostate cancer and improves disease-free survival in combination with radiation therapy in patients with locally advanced disease, but has been associated with bone loss and increased fracture risk. Fractures are an important contributor to morbidity associated with androgen deprivation therapy. Existing therapies have shown prevention of bone loss, but have yet to demonstrate reduction in fractures. Denosumab, a human monoclonal antibody that inhibits receptor activator for nuclear factor- $\mathrm{KB}$ ligand, impacts osteoclasts formation and survival, and has been shown to increase bone density

D. S. Silver $(\bowtie)$

Cedars Sinai Medical Center/UCLA School of Medicine and the OMC Clinical Research Center,

8641 Wilshire Boulevard, Suite 301,

Beverly Hills, CA 90211, USA

e-mail: davids@OMCresearch.org in postmenopausal osteoporosis and other hormone deprivation states.

\begin{abstract}
Aims
The study was designed to examine whether administration of denosumab in men with nonmetastatic prostate cancer would affect bone mineral density and rates of fracture in the vertebral spine and hip in a randomized, double-blind, multicenter, placebo-controlled trial.
\end{abstract}

\section{Methods}

Patients with advanced local prostate cancer who were expected to be on androgen deprivation therapy (bilateral orchiectomy or gonadotropin-releasing hormone-agonist therapy) for at least 12 months of the study period were considered for inclusion in the trial. If under 70 years of age, T-score at the lumbar spine, total hip, or femoral neck had to be less than -1.0 at baseline or they must have had a previous osteoporotic fracture. They could not have taken an oral bisphosphonate within 1 year prior to entering the trial or ever had exposure to an intravenous bisphosphonate. A total of 734 patients were assigned to each group and all were instructed to take appropriate amounts of calcium and vitamin D. The primary end point was percent change in bone mineral density of the lumbar spine at 24 months. Secondary end points included percent change in bone mineral density at 36 months in the vertebral spine, percent change in the total hip and femoral neck at 24 and 36 months, incidence of newly diagnosed vertebral fracture at 36 months, fracture at any site, time to first fracture, and safety events. The study was originally intended to last 24 months but was extended to 
36 months specifically to further examine the fracture prevention end point.

\section{Results}

A total of 912 (62\%) of 1468 patients completed the 36-month study, with the primary reason for discontinuation being withdrawal of consent when the blinded period was extended from 24 to 36 months. A similar number discontinued study in the placebo and treatment groups. Mean age was 75 years, and most patients had been on androgen deprivation therapy for greater than 6 months at initiation of study. Increased bone mineral density was noted at all sites. The primary end pointchange in bone density at the lumbar spine at 24 months - was achieved, increasing $5.6 \%$ in the denosumab group versus $-1.0 \%$ in the placebo group $(P<0.001)$. Changes in bone mineral density in the lumbar spine were statistically significantly different as early as 1 month on therapy at the lumbar spine. At all other sites (total hip, femoral neck, distal third of radius, and total body), statistically significant increases in bone density on denosumab when compared with placebo were observed. There was no difference in various predetermined subgroups that were analyzed. Denosumab-treated patients had decreases in vertebral fractures observed at 12,24 , and 36 months. The 36month cumulative incidence of fracture was $3.9 \%$ in placebo-versus $1.5 \%$ in denosumab-treated patients (CI, $0.19-0.78 ; P=0.006)$. Fracture at any site was diminished but did not reach statistical significance $(P=0.10)$. No difference in time to first fracture was noted. Bone turnover markers decreased significantly on denosumab when compared with placebo. Cataracts appeared more prevalent in the denosumab group. Serious infections were noted in $5.9 \%$ in the denosumab patients compared with $4.6 \%$ on placebo. A total of $6 \%$ of patients died in each group during the study. No cases of delayed healing of nonvertberal fractures or osteonecrosis of the jaw were reported.

\section{Discussion}

This is the first trial to demonstrate reduction in vertebral fracture in men on androgen deprivation therapy with an antiresorptive agent. Several studies have shown significant increases in bone density, but were not powered or failed to demonstrate fracture efficacy [1-5]. This trial was not powered to answer the question of nonvertebral fractures, although the data suggested a trend to reduction in all fractures. In addition, the effects in terms of bone mineral density were seen early on, as soon as 1 month on therapy, and although time to first fracture was not reduced, the small number of patients who fractured in the first month on denosumab (only two) may have impacted that analysis. There were no concerning safety signals noted, specifically no significant increased risk of infection and no cases of osteonecrosis, which has reportedly been seen in higher incidence in the cancer patient population on bisphosphonates.

The study did not examine men with metastatic disease who might be at even higher risk for fracture with androgen therapy, although another recently published study suggested that use of denosumab reduced the rate of skeletalrelated events in patients with metastatic disease, even if they had already failed a bisphosphonate [6]. Although the rate of bone loss and the incidence of vertebral fracture in men on androgen deprivation therapy for prostate cancer are not well characterized, bone loss in this group is universally recognized. No approved therapy has been established for the treatment of bone loss in men with prostate cancer. No direct comparison with other treatments, such as bisphosphonates of selective estrogen receptor modulators, has been performed; thus, determining which therapy is most effective cannot be concluded from this trial.

\section{Comments}

Denosumab is the first drug shown to reduce vertebral fractures in men on androgen deprivation therapy for prostate cancer. Effects on bone mineral density are seen very early on, as soon as 1 month into therapy. With the increasing incidence of prostate cancer, more frequent use of androgen deprivation therapy, and increased longevity in men with disease, twice-yearly administration of denosumab appears to be a safe and effective methodology of preventing one the most common morbidities seen in men with prostate cancer.

Disclosure Dr. David S. Silver is on the speakers' bureau of Genetech and GlaxoSmithKline.

Open Access This article is distributed under the terms of the Creative Commons Attribution Noncommercial License which permits any noncommercial use, distribution, and reproduction in any medium, provided the original author(s) and source are credited.

\section{References}

1. Smith MR, McGovern FJ, Zietman AL, et al: Pamidronate to prevent bone loss during androgen-deprivation therapy for prostate cancer. N Engl J Med 2001, 345:948-955.

2. Michaelson MD, Kaufman DS, Lee H, et al.: Randomized controlled trial of annual zoledronic acid to prevent gonadotropin- 
releasing hormone agonist-induced bone loss in men with prostate cancer. J Clin Oncol 2007, 25:1038-1042.

3. Smith MR, Eastham J, Gleason DM, et al.: Randomized controlled trial of zoledronic acid to prevent bone loss in men receiving androgen deprivation therapy for nonmetastatic prostate cancer. $\mathbf{J}$ Urol 2003, 169:2008-2012.

4. Greenspan SL, Nelson JB, Trump DL, Resnick NM: Effect of once-weekly oral alendronate on bone loss in men receiving androgen deprivation therapy for prostate cancer: a randomized trial. Ann Intern Med 2007, 146:416-424.
5. Smith MR, Fallon MA, Lee H, Finkelstein HS: Raloxifene to prevent gonadotropin-releasing hormone agonist-induced bone loss in men with prostate cancer: a randomized controlled trial. J Clin Endocrinol Metab 2004, 89:38413846.

6. Fizazi K, Lipton A, Mariette X, et al.: Randomized phase II trial of denosumab in patients with bone metastases from prostate cancer, breast cancer, and other neoplasms after intravenous bisphosphonates. J Clin Oncol 2009, 27:15641571 . 